\title{
The Short-Term Effects of Difference Frequency of Transcutaneous Electrical Nerve Stimulation on Pain Relief using c-fos Expression in Spinal Cord with Knee Osteoarthritis Rats
}

\author{
Hyun-Mo Koo, PT, PhD $\cdot$ Sang-Su Na, PT, PhD ${ }^{1 \dagger}$ \\ Department of Physical Therapy, College of Science, Kyungsung University \\ ${ }^{1}$ Department of Physical Therapy, Daegu University
}

Received: July 29, 2016 / Revised: July 29, 2016 / Accepted: August 23, 2016

(c) 2016 J Korean Soc Phys Med

\begin{abstract}
| Abstract |
PURPOSE: The purpose of this study was to determine the effect of different frequencies $(4 \mathrm{~Hz}$ and $100 \mathrm{~Hz})$ of transcutaneous electrical nerve simulation (TENS) on pain relief using c-fos expression in the spinal cord of rat osteoarthritis to investigate the appropriate frequency for pain relief.
\end{abstract}

METHODS: Total of 30 Sprague-Dawley rats was used and randomly divided 2 groups according TENS frequency and applicate the TENS during 3 period ( 3 days, 7 days, 10 days). The induction of osteoarthritis by $3 \mathrm{mg}$ monosodium iodoacetat was injected into the right knee joint of rats. Three days later, commercially available TENS unit was used for stimulation was set to 20 minutes on 3, 7, 10 days after surgery. Western blot analysis system was used to detect immunoreactive proteins. The thickness of the bands were photographically measured by Scion Image.

RESULTS: When investigating the c-fos expression of

†Corresponding Author : deshitart@naver.com

This is an Open Access article distributed under the terms of the Creative Commons Attribution Non-Commercial License (http://creativecommons.org/licenses/by-nc/3.0) which permits unrestricted non-commercial use, distribution, and reproduction in any medium, provided the original work is properly cited.
TENS on spinal cord in OA knee over 10 days, between-groups differences in c-fos expression reached a significant level by day 10 . For within-groups comparisons, the c-fos expression decreased significantly across days in low- and high-frequency TENS groups.

CONCLUSION: Whether at low- and high-frequency, the TENS as a therapy obtained beneficial effects of pain relief and TNES at high-frequency is more beneficial effects on the pain relief when TENS applied at injury site.

Key Words: Frequencies, Osteoarthritis, Transcutaneous electrical nerve stimulation, Pain relief

\section{Introduction}

Osteoarthritis $(\mathrm{OA})$ is the most common degenerative joint musculoskeletal disease that causes physical disability (Hinton et al., 2002). Mechanical stress and inflammation are involved in $\mathrm{OA}$ onset and progression. Disease management of $\mathrm{OA}$ is benefited in the early stage by therapies such as exercise, diet, anti-inflammatory medicines, and injections that delay the disease's progression and pain. 
Pain is one of the major factors in the interruption of movement, so pain relief is important for the quality of life for OA patients. Reports of pain in humans and animals have demonstrated that nociceptive stimuli can facilitate metabolic changes on the dorsal horn of the spinal cord (Klein et al., 2004; Schadrack et al., 1999). Met- and leu-enkephalin peptides are found on primary afferent terminals in the spinal cords in rodents (Wang et al., 2010). The encephalin can reduce the responses of primary afferent stimulation, particularly of activated neurons, by noxious stimuli located in the dorsal horn (Duggan et al., 1977). This is caused by inhibiting transmitter release by the nociceptive primary afferents (Beaudry et al., 2011). Thus, spinal nociception may modulate encephalin by inhibiting the primary afferents and subsequently by inhibiting the spinal nociceptive neurons. C-fos is a general pain marker in the spinal cord and is known as the suppressor of encephalin. Previous studies showed that c-fos activated neurons containing preproenkephalin (PPE), which produces encephalin and encodes for the proenkephalin polypeptide that yields methionine (met) and leucine (leu-) encephalin peptides after different types of nociceptive inputs (Hossaini et al., 2014).

Transcutaneous electrical nerve stimulation (TENS) is one of the physical modalities most often used clinically for the treatment of many types of pain, including that caused by OA of the knee. TENS is well documented in relieving pain, and various simulation parameters have been adopted, from $4 \mathrm{~Hz}$ to $100 \mathrm{~Hz}$ frequencies (Law and Cheing, 2004; Lee and Song, 2013). Previous studies found that both high-frequency TENS $(100 \mathrm{~Hz})$ and low-frequency TENS $(4 \mathrm{~Hz})$ affected hyperalgesia after treatment, and biochemical studies have demonstrated that different frequencies of TENS activate different endogenous opioid systems in the central nervous system (CNS) (Andersson et al., 1977; Han et al., 1991; Sluka et al., 1998). High-frequency stimulation accelerates the release of dynorphin, while low-frequency stimulation releases enkephalins, B-endorphins, and endomorphins, which act on different receptors in the CNS. However, there is conflicting evidence regarding the effect of TENS frequencies. Previous study have demonstrated that high-frequency TENS produces more effective analgesia and a greater decrease in the activity of dorsal horn neurons than does low-frequency TENS (Willer et al., 1982). However, evidence that low-frequency TENS provides a greater reduction of the activity of spinothalamic tract cells has also been reported (Garrison and Foreman, 1994; Lee et al., 1985).

Thus, the purpose of this study was to determine the effect of different frequencies $(4 \mathrm{~Hz}$ and $100 \mathrm{~Hz})$ of TENS on pain relief using c-fos expression in the spinal cord of rat osteoarthritis models.

\section{Methods}

\section{Experimental animals and Procedures}

In this study, total 30 adult male Wistar rats (8 weeks of age) weighting between 250 and $300 \mathrm{~g}$. The induction of osteoarthritis was performed as previously reported (Jekal et al., 2014). In brief, 3mg monosodium iodoacetat (MIA, Sigma, St Louis, MO, America) was injected into the right knee joint of rats. Three days later, commercially available TENS unit was used for stimulation was set to 20 minutes per day for 3, 7, 10 days after surgery. Two frequencies were used, a high-frequency $(100 \mathrm{~Hz} ; 20-40 \mathrm{~mA})$ and a low-frequency $(4 \mathrm{~Hz} ; 10-20 \mathrm{~mA})$ using pairs of rubber electrodes placed on right knee. Pulse duration was either $100 \mu \mathrm{sec}$ or $250 \mu \mathrm{sec}$. Injected animals were divided 2 groups randomly according to TENS (Endomed, Enraf-nonius, Netherlands) frequency and applicate the TENS during 3 period: high-frequency TENS $(100 \mathrm{~Hz})$ for 3 days after surgery, high-frequency TENS $(100 \mathrm{~Hz})$ for 7 days after surgery, high-frequency TENS $(100 \mathrm{~Hz})$ for 10 days after surgery, low-frequency TENS $(4 \mathrm{~Hz})$ for 3 
The Short-Term Effects of Difference Frequency of Transcutaneous Electrical Nerve Stimulation on Pain Relief using c-fos Expression in Spinal Cord with Knee Osteoarthritis Rats | 51

Table 1. The statistical analysis of c-fos expression level in each group

\begin{tabular}{cccccc}
\hline Subjects & SS & Df & MS & F & P \\
\hline Frequency & 5400.40 & 2 & 2700.70 & 569.26 & $.00^{*}$ \\
Period & 145.53 & 1 & 145.53 & 30.71 & $.00^{*}$ \\
Frequency / period & 68.27 & 2 & 34.63 & 7.21 & $.00^{*}$ \\
\hline
\end{tabular}

$* \mathrm{P}<.05$

days after surgery, low-frequency TENS (4Hz) for 7 days after surgery, low-frequency TENS (4Hz) for 10 days after surgery.

\section{Western blot analysis}

After the animals were anesthetized and sacrificed, the spinal cord of each group were collected and then homogenized and lysed with RIPA buffer. Equal amount of protein $(20 \mu \mathrm{g})$ were resolve via $12 \%$ sodium dodecyl sulfate-polyacrylamide gel electrophoresis (SDS-PAGE) and transferred to nitrocellulose membranes. The blots was washed with TBS-T, blocked with 5\% skim milk for 1 hour, then incubated with anti c-fos (1:1000) antibody at the dilutions recommended by the suppliers. The membranes was washed and the primary antibody was detected using horseradish peroxidase-conjugated goat anti-mouse IgG (1:3000). Western blot analysis system was used to detect immunoreactive proteins. The thickness of the bands were photographically measured by Scion Image.

\section{Statistical analysis}

PASW 18 was used for the analysis and significance level was set at .05. Repeated two-way ANOVA was used to analyze the effects of the group and period on the c-fos expression level, and Fisher's LSD test was used for post hoc evaluations.

\section{Results}

1. Comparison of $\mathrm{c}-$ fos expression level between low- and high-frequency TENS group

The c-fos expression level of frequency and period in each group was two-way repeated ANOVA and LSD test was used for the post hoc evaluations. When investigating the c-fos expression of TENS on spinal cord in OA knee pain over 10 days, significant interaction between the 'frequency' and 'period' was observed $(\mathrm{p}=.004)$ (Table 1). The between-groups differences in c-fos expression reached

Table 2. Changes of c-fos expression level on the 2 groups across period

\begin{tabular}{ccccc}
\hline Frequency / period & 3 days & 7 days & 10 days & $\begin{array}{c}\text { Within group } \\
(\mathrm{p} \text {-value })\end{array}$ \\
\hline L-TENS & $16383.80 \pm 592.91$ & $8799.60 \pm 568.79 \dagger$ & $6118.20 \pm 412.12^{\dagger \ddagger}$ & $.00^{*}$ \\
H-TENS & $14663.60 \pm 1228.05$ & $8603.80 \pm 540.52^{\dagger}$ & $4432.80 \pm 291.28^{\dagger \ddagger} \oint$ & $.00^{*}$ \\
$\begin{array}{c}\text { Between group } \\
\text { (p-value) }\end{array}$ & .09 & .76 & $.00^{*}$ & 8.44 \\
$\mathrm{~T}$ & 2.26 & .32 & .44 \\
\hline
\end{tabular}

Mean $\pm \mathrm{SD} ;$ mean \pm standard deviation

$t=$ significant difference from 3 day $\mathrm{P}<.05$

$\ddagger=$ significant difference from 7 day $\mathrm{P}<.05$

$\oint=$ significant difference from L-TENS $\mathrm{P}<.05$

L-TENS: low-intensity transcutaneous electrical nerve simulation

H-TENS: high-intensity transcutaneous electrical nerve simulation 


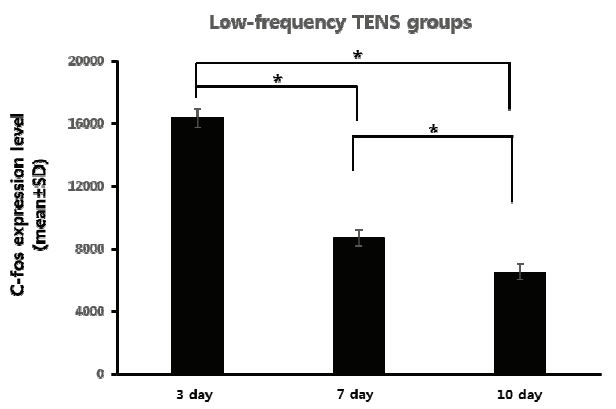

Fig. 1. C-fos expression level of low-frequency TENS group

a significant level by day $10(\mathrm{p}=.001)$. However, the $\mathrm{c}$-fos expression level were not significantly different among the low- and high-frequency groups in day 3, day 7 (Table 2).

\section{Comparison of $\mathrm{c}-$ fos expression level within- groups}

For within-groups comparisons, the c-fos expression decreased significantly across days in high-and lowfrequency TENS groups. From day 3 to day 10, there was $63.7 \%$ decrease in low-frequency TNES group (Fig. 1), $69.8 \%$ decrease in high-frequency TENS groups (Fig. 2).

\section{Discussion}

$\mathrm{A}$ is a chronic disease that is associated with reduced muscle strength, disability, and a progressive loss of function due to pain (Hinman et al., 2002). The progression of pain is not fully understood, but recent studies suggest that the central and peripheral nervous systems may be involved in chronic pain due to changes in the excitability of the spinal pathway (Imamura et al., 2008). Thus, the control of pain could influence muscle activation and normal movement for patients with OA. This study investigated the pain levels using c-fos in the spinal cord using Western blot analysis.

From the study results, it is evident that high- and

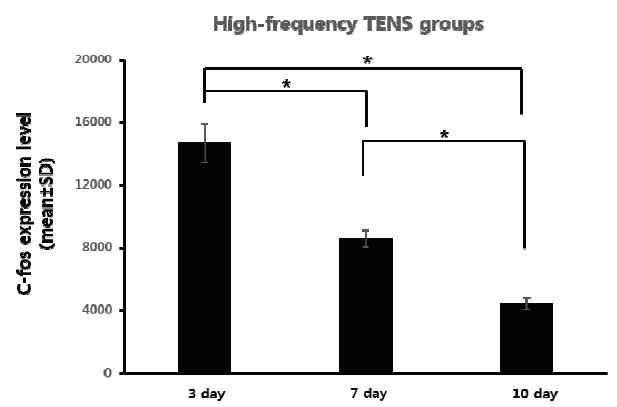

Fig. 2. C-fos expression level of high-frequency TENS group

low-frequency TENS reduces c-fos expression in the spinal cords of rats with osteoarthritis after treatment. TENS is often used clinically to treat pain and is widely applied to patients with osteoarthritis. Many studies have been done to find the most effective parameters, including the frequency of TENS, but these parameters have yet to be identified. Ericksson and colleagues showed that low frequencies and high frequencies are more effective for the relief of pain compared with conventional TENS, but a different study demonstrated that there were no significant differences in pain levels (Eriksson et al., 1984; Jensen et al., 1991). Mechanical studies for the frequency of TENS showed various analgesic mechanisms in which lowfrequency stimulation increases met-enkephalin-Arg- Phe (MEAP), while high-frequency stimulation accelerates the dynorphin (Han et al., 1991). Another study showed that alternating the stimulation frequency of TENS between low $(2 \mathrm{~Hz})$ and high $(100 \mathrm{~Hz})$ frequencies produces a synergistic interaction of dynorphin and encephalin and that it has more analgesic effect than a fixed frequency (Xiao-Hong et al., 1994). Following these results, different frequencies of TENS seem to relate to different analgesic mechanisms.

In this study, repeated application of low- and high-frequency TENS showed a significant decrease of c-fos expression. This is supported by the findings of the previous study, in which the application of TENS was useful for pain relief (Hossaini et al., 2014). The findings 
of this present study also showed that high-frequency TENS produced significant $\mathrm{c}-$ fos expression over 10 days in group compared with low-frequency TENS in another group.

Hyperalgesia, an increased response to noxious stimuli, consists of primary hyperalgesia that occurs at the site of injury and secondary hyperalgesia that occurs outside the site of injury (Willis Jr and Coggeshall, 2012). The TENS has an effect on primary and secondary hyperalgesia induced by joint inflammation. Previous study has shown that only high-frequency TENS significantly reduces primary hyperalgesia and has suggested that the nociceptive processing of primary and secondary hyperalgesia results from different mechanisms, so different frequencies are only important with respect to reducing primary hyperalgesia (King and Sluka, 2001). The current study demonstrated that high-frequency TENS more reduce the c-fos expession level than low-frequency TENS in 10 day. It is supported by the finding of the pervious study, high-frequency TENS should produce better pain relief when applied at the site of injury (Priya and Kathleen, 2000). In the same manner, this study directly applied the TENS at injected knee so high-frequency TENS may be show more reduce of pain relief in 10 day.

Thus, the current study demonstrated that low- and high-frequency TENS reduces the c-fos expression in the spinal cord and that this may be related to the hyperalgesia mechanism. Therefore, the application of low- and high-frequency TENS may produce pain relief in a cumulative manner from day 3 to day 10 . But in the current study was examined without taking intensity or pulse duration into account. Although pulse duration and intensity alone may not be key factors in the inhibition produced by TENS, no correlation with c-fos expression was found, so it is difficult to the c-fos expression was the result of only frequency. Further study is necessary investigate the pulse duration and intensity with respect to c-fos expression in an animal OA model.

\section{Conclusion}

In the present study, TENS was applied to rats with $\mathrm{OA}$ in order to investigate the appropriate frequency for pain relief. According to c-fos expression level measured by western blot analysis, in low- and high- frequency groups, c-fos expression level showed significant decreased over 10 days. Therefore, whether at low- or high-frequency, the TENS as a therapy obtained beneficial effects of pain relief and TENS at high-frequency is more beneficial effects on the pain relief when TENS applied at injury site.

\section{Acknowledgements}

This research was supported by Kyungsung University Research Grants in 2016.

\section{References}

Andersson S, Holmgren E, Roos A. Analgesic effects of peripheral conditioning stimulation-II. Importance of certain stimulation parameters. Acupunct Electrother Res. 1977;2(3-4):237-46.

Beaudry H, Dubois DGL. Activation of spinal $\mu$-and $\delta$-opioid receptors potently inhibits substance $P$ release induced by peripheral noxious stimuli. J Neurosci. 2011;31(37):13068-77.

Duggan A, Hall JP. Enkephalins and dorsal horn neurones of the cat: effects on responses to noxious and innocuous skin stimuli. Br J Pharmacol. 1977;61(3): 399-408.

Eriksson MB, Sjölund BH, Sundbärg G. Pain relief from peripheral conditioning stimulation in patients with chronic facial pain. J Neurosurg. 1984;61(1):149-55.

Garrison DW, Foreman RD. Decreased activity of spontaneous and noxiously evoked dorsal horn cells during 
transcutaneous electrical nerve stimulation (TENS). Pain. 1994;58(3):309-15.

Han J, Chen X, Sun S, et al. Effect of low-and high-frequency TENS on Met-enkephalin-Arg-Phe and dynorphin A immunoreactivity in human lumbar CSF. Pain. 1991;47(3):295-8.

Hinman RS, Bennell KL, Metcalf BR, et al. Delayed onset of quadriceps activity and altered knee joint kinematics during stair stepping in individuals with knee osteoarthritis. Arch Phys Med Rehabil. 2002;83(8): 1080-6.

Hinton R, Moody RL, Davis AW, et al. Osteoarthritis: diagnosis and therapeutic considerations. Am Fam Physician. 2002;65(5):841-8.

Hossaini M, Duraku LS, Kohli SK, et al. Spinal distribution of c-Fos activated neurons expressing enkephalin in acute and chronic pain models. Brain Res. 2014;1543:83-92.

Imamura M, Imamura ST, Kaziyama $\mathrm{HH}$, et al. Impact of nervous system hyperalgesia on pain, disability, and quality of life in patients with knee osteoarthritis: a controlled analysis. Arthritis Care Res (Hoboken). 2008;59(10):1424-31.

Jekal SJ, Kwon PS, Kim JK, et al. Effect of $840 \mathrm{~nm}$ Light-Emitting Diode (LED) Irradiation on Monosodium Iodoacetate-Induced Osteoarthritis in Rats. J Korean Soc Phys Med. 2014;9(2):151-9.

Jensen H, Zesler R, Christensen T. Transcutaneous electrical nerve stimulation (TNS) for painful osteoarthrosis of the knee. Int J Rehabil Res. 1991;14(4):356-58.

King EW, Sluka KA. The effect of varying frequency and intensity of transcutaneous electrical nerve stimulation on secondary mechanical hyperalgesia in an animal model of inflammation. J Pain. 2001;2(2):128-33.

Klein T, Magerl W, Hopf HC, et al. Perceptual correlates of nociceptive long-term potentiation and long-term depression in humans. J Neurosci. 2004;24(4):96471.

Law P, Cheing G. Optimal stimulation frequency of transcutaneous electrical nerve stimulation on people with knee osteoarthritis. J Rehabil Med. 2004;36(5): 220-5.

Lee KH, Chung JM, Willis Jr WD. Inhibition of primate spinothalamic tract cells by TENS. J Neurosurg. 1985;62(2):276-87.

Lee SH, Song CH. The Effects of Transcutaneous Electrical Nerve Stimulation (TENS) on the Neuropathic Pain in Peripheral Nerve Injury. J Korean Soc Phys Med. 2013;8(1):79-89.

Prita G, Kathleen AS. Effect of varying frequency, intensity, and pluse duration of transcutaneous electrical nerve simulation on primary hyperalgesia in inflamed rats. Arch Phys Med Rehabil. 2000;81:984-90.

Schadrack J, Neto F, Ableitner A, et al. Metabolic activity changes in the rat spinal cord during adjuvant monoarthritis. Neuroscience. 1999;94(2):595-605.

Sluka K, Bailey K, Bogush J, et al. Treatment with either high or low frequency TENS reduces the secondary hyperalgesia observed after injection of kaolin and carrageenan into the knee joint. Pain. 1998;77(1): 97-102.

Wang HB, Zhao B, Zhong YQ, et al. Coexpression of $\delta$-and $\mu$-opioid receptors in nociceptive sensory neurons. Proc Natl Acad Sci USA. 2010;107(29):13117-22.

Willer JC, Roby A, Boulu P, et al. Comparative effects of electroacupuncture and transcutaneous nerve stimulation on the human blink reflex. Pain. 1982;14(3):267-78.

Willis Jr WD, Coggeshall RE. Sensory Mechanisms of the Spinal Cord: Volume 1 Primary Afferent Neurons and the Spinal Dorsal Horn. Texas. The University of Texas Medical Branch Galveston. 2012.

XiaoHong C, SuFong G, ChungGwo C, et al. Optimal conditions for eliciting maximal electroacupuncture analgesia with dense-and-disperse mode stimulation. Am J Acupunct. 1994;22(1):47-53. 